\title{
Human stem cell models to study host-virus interactions in the central nervous system
}

\section{Oliver Harschnitz $\mathbb{B}^{1,2 \otimes}$ and Lorenz Studer $\mathbb{B}^{1,2}$}

Abstract | Advancements in human pluripotent stem cell technology offer a unique opportunity for the neuroimmunology field to study host-virus interactions directly in disease-relevant cells of the human central nervous system (CNS). Viral encephalitis is most commonly caused by herpesviruses, arboviruses and enteroviruses targeting distinct CNS cell types and often leading to severe neurological damage with poor clinical outcomes. Furthermore, different neurotropic viruses will affect the CNS at distinct developmental stages, from early prenatal brain development to the aged brain. With the unique flexibility and scalability of human pluripotent stem cell technology, it is now possible to examine the molecular mechanisms underlying acute infection and latency, determine which CNS subpopulations are specifically infected, study temporal aspects of viral susceptibility, perform high-throughput chemical or genetic screens for viral restriction factors and explore complex cell-non-autonomous disease mechanisms. Therefore, human pluripotent stem cell technology has the potential to address key unanswered questions about antiviral immunity in the CNS, including emerging questions on the potential CNS tropism of severe acute respiratory syndrome coronavirus 2 (SARS-CoV-2).

Embryonic stem cells Pluripotent stem cells that are capable of giving rise to all lineages, but not to extraembryonic tissues. They are derived from the inner cell mass of the developing embryo.
The isolation of human embryonic stem cells ${ }^{1}$ and transcription factor-based reprogramming of somatic cells into human induced pluripotent stem cells (iPSCs) ${ }^{2}$ are important breakthroughs in the stem cell field. The directed differentiation of human pluripotent stem cells (hPSCs; a term referring to both human embryonic stem cells and human iPSCs) along the three germ layers enables the derivation, study and perturbation of potentially any human cell type. Key applications of hPSCs include the study of human development ${ }^{3-7}$, modelling human disease $^{8-11}$ and the development of cell-based therapies in regenerative medicine ${ }^{12-18}$. Past human studies on neurological and neuroimmunological disease relied on autopsy or biopsy materials, the isolation of cerebrospinal fluid or peripheral blood samples. The lack of routine access to primary human central nervous system (CNS) cell types has precluded most mechanistic studies, prevented the establishment of robust in vitro disease models and made it impossible to perform high-content chemical or genetic screens directly in human cells. Therefore, hPSC technology offers a particularly attractive approach for studying CNS disease and is an ideal tool to probe neuroimmunological disorders.

Animal models have yielded important insights into the physiology and dysfunction of the mammalian immune system. Animal models can be genetically altered, and disease phenotypes can be studied within an entire organism to dissect complex interactions between organ systems and diverse cell types. However, there are considerable species-specific differences of the immune system that complicate the translation of findings in animal models to human disease. General differences in metabolism and cell turnover may play a role, but species-specific differences of the immune system are also profound. Comparing mice and humans, we find key differences in many immune components, including in the cellular composition of leukocytes, in signalling components of $\mathrm{B}$ cells and $\mathrm{T}$ cells, in Toll-like receptors (TLRs), in cytokines and cytokine receptors and in immunoglobulin class switching ${ }^{19,20}$. In the brain, microglia show species-specific divergence at physiological levels (for example, in proliferation and in the expression of sialic acid-binding immunoglobulin-like lectins (SIGLECs)) and in their neuroinflammatory and disease states (for example, responses to transforming growth factor- $\beta$ (TGF $\beta$ ) signalling in models of Alzheimer disease $)^{21}$. The use of hPSC-derived cell types provides a tractable human in vitro disease platform for mechanistic studies, for identifying novel therapeutic targets and for translating targets into compounds 


\section{Induced pluripotent \\ stem cells \\ (iPSCs). Pluripotent stem cells derived from terminally differentiated somatic cells through cellular reprogramming via the transient expression of a defined set of transcription factors.}

Directed differentiation The guided differentiation of pluripotent stem cells towards a desired somatic cell state using morphogens and small molecules.

Cerebellar granule cell neurons

The most numerous neurons in the human brain. They are found in the thick granular layer of the cerebellar cortex.

\section{Purkinje neurons}

A class of GABAergic neurons located within the Purkinje layer of the cerebellum. They arise from progenitors in the ventricular neuroepithelium of the cerebellar primordium during embryogenesis. for therapeutic intervention. In addition to capturing the patient's genetic information via iPSC technology, powerful genome editing tools are used routinely in hPSCs to genetically correct or induce the expression of disease-linked alleles.

To date, hPSC technology has been mostly applied to neurodevelopmental and neurodegenerative disorders, such as microcephaly ${ }^{22}$, spinal muscular atrophy ${ }^{23}$, familial dysautonomia ${ }^{24}$, amyotrophic lateral sclerosis ${ }^{8}$, Alzheimer disease ${ }^{9}$ and Parkinson disease ${ }^{25}$. However, hPSC technology is also increasingly used to study autoimmune-induced or virus-induced diseases of the nervous system ${ }^{26}$. Such studies have examined how autoantibodies target neuronal epitopes leading to neuronal dysfunction that closely recapitulates clinical phenotypes and treatment responsiveness ${ }^{27-29}$. Studies on host-virus interactions in hPSC-derived neural lineages include work on herpes simplex virus 1 (HSV-1) and Zika virus (ZIKV), which affect distinct subpopulations, such as postmitotic neurons versus neural progenitor cells (NPCs), providing important insights into virus-induced CNS pathophysiology $\mathrm{y}^{30-33}$. This Review presents an overview of hPSC-based studies of CNS host-virus interactions and explores how this technology can address key outstanding questions in the neuroimmunology field. In the final section of the Review, we discuss how such studies may also help to address emerging questions concerning CNS involvement and neurological complications in severe acute respiratory syndrome coronavirus 2 (SARS-CoV-2) infection.

\section{Using hPSCs to model CNS-resident cell types} CNS-resident cells show differential susceptibility to viral infection. The brain comprises numerous neuronal and glial cell types that are essential for CNS function. The extensive cellular heterogeneity in the CNS entails vast heterogeneity in cell type-specific immunological responses. Astrocytes and microglia play key roles in antiviral immunity, and neurons themselves are not mere bystanders. Neurons exhibit innate antiviral immune responses and produce various interferons and express interferon-stimulated genes in response to viral infection $^{34,35}$. Selective vulnerability is a frequent, albeit poorly understood, characteristic of neurological disorders, such as the motor neuron degeneration seen in amyotrophic lateral sclerosis or the loss of dopaminergic neurons in Parkinson disease. Selective vulnerability is similarly seen in viral encephalitis as illustrated for West Nile virus infection, where autopsy reports describe a lack of viral infection in cerebellar granule cell neurons, while Purkinje neurons and cortical neurons are highly susceptible $^{36}$. Such differential West Nile virus permissivity in granule cell versus cortical neurons involves higher basal levels of key interferon-stimulated genes (Ifi27, Irg1 (also known as Acod1) and Rsad2) and a more potent interferon-stimulated gene response following interferon- $\beta$ (IFN $\beta$ ) stimulus ${ }^{37}$. In patients with herpes simplex encephalitis, the frontotemporal regions of the cerebral cortex are most commonly affected $^{38,39}$. By contrast, patients with DBR1 deficiency display a brainstem encephalitis, caused by multiple neurotropic viruses, including HSV-1 (REF. ${ }^{40}$ ). Finally,
ZIKV preferentially targets NPCs and microglia, leading to impaired prenatal brain development resulting in microcephaly ${ }^{41,42}$. In addition to direct CNS tropism, viral infections can lead to systemic inflammatory responses that may harm CNS-resident cell types either directly or through migration of immune cells into the $\mathrm{CNS}^{43}$. One such example is the development of postencephalitic parkinsonism that followed the influenza pandemic in 1918. The cause of the resulting parkinsonism remains unclear, with several potential mechanisms proposed, such as chronic CNS inflammation following acute viral infection, invasion of peripheral immune cells into the CNS following loss of integrity of the blood-brain barrier (BBB) or damage due to postinfectious autoimmunity ${ }^{44,45}$.

Why certain neuronal or glial cell types show resistance or susceptibility to a given virus remains largely unknown. To probe cell type-specific innate immune responses, it is imperative to obtain purified neural lineages on demand. The derivation of highly enriched CNS subtypes from hPSCs allows modelling of selective vulnerabilities in vitro and the identification of cell type-specific innate immune mechanisms. Furthermore, by combining several distinct cell types in co-culture systems, either in two or three dimensions, one can study the role of cell-non-autonomous signalling in antiviral immunity $^{46}$. Those may include anti-inflammatory mechanisms during the acute phase ${ }^{47,48}$, but also proinflammatory cascades leading to chronic inflammation and long-term neurological deficits ${ }^{43,49-51}$. Systemic inflammatory signals that negatively impact CNS function, such as virus-induced brain endothelial cell inflammation ${ }^{52}$ or T cell-mediated synaptic pruning ${ }^{43}$, could also be modelled with hPSC technology by establishing relevant co-culture systems of neural and non-neural cell types.

Neural induction and differentiation of hPSCs. Sophisticated differentiation strategies have been developed to yield region-specific neuronal subtypes ${ }^{12,35,53-60}$, astrocytes ${ }^{61-64}$ and oligodendrocytes ${ }^{65}$. The first step of in vitro neural differentiation mimics the developmental process of neural induction during the gastrula stage ${ }^{66}$ (FIG. 1). Inhibition of bone morphogenetic protein (BMP) signalling - and/or activation of fibroblast growth factor (FGF) signalling - combined with nodal inhibition suppresses non-neural, extraembryonic and mesodermal/ endodermal fates and enables neural differentiation. This strategy is implemented in a widely used method ('dual-SMAD inhibition') ${ }^{54}$ by concomitant treatment with the TGF $\beta$ inhibitor SB431542 and the BMP inhibitor noggin (or LDN193189), a method that forms the basis of many neural differentiation paradigms today.

Regional specification similarly mimics in vivo development where morphogen gradients drive patterning in the anterior-posterior (FGFs, WNTs and retinoic acid) and dorsal-ventral (WNTs, BMP and sonic hedgehog $(\mathrm{SHH})$ ) axis. Exposure to specific combinations of morphogens at distinct time points of differentiation results in the derivation of diverse neuronal lineages from hPSCs, ranging from forebrain cortical, midbrain dopaminergic and hindbrain neurons to spinal cord 


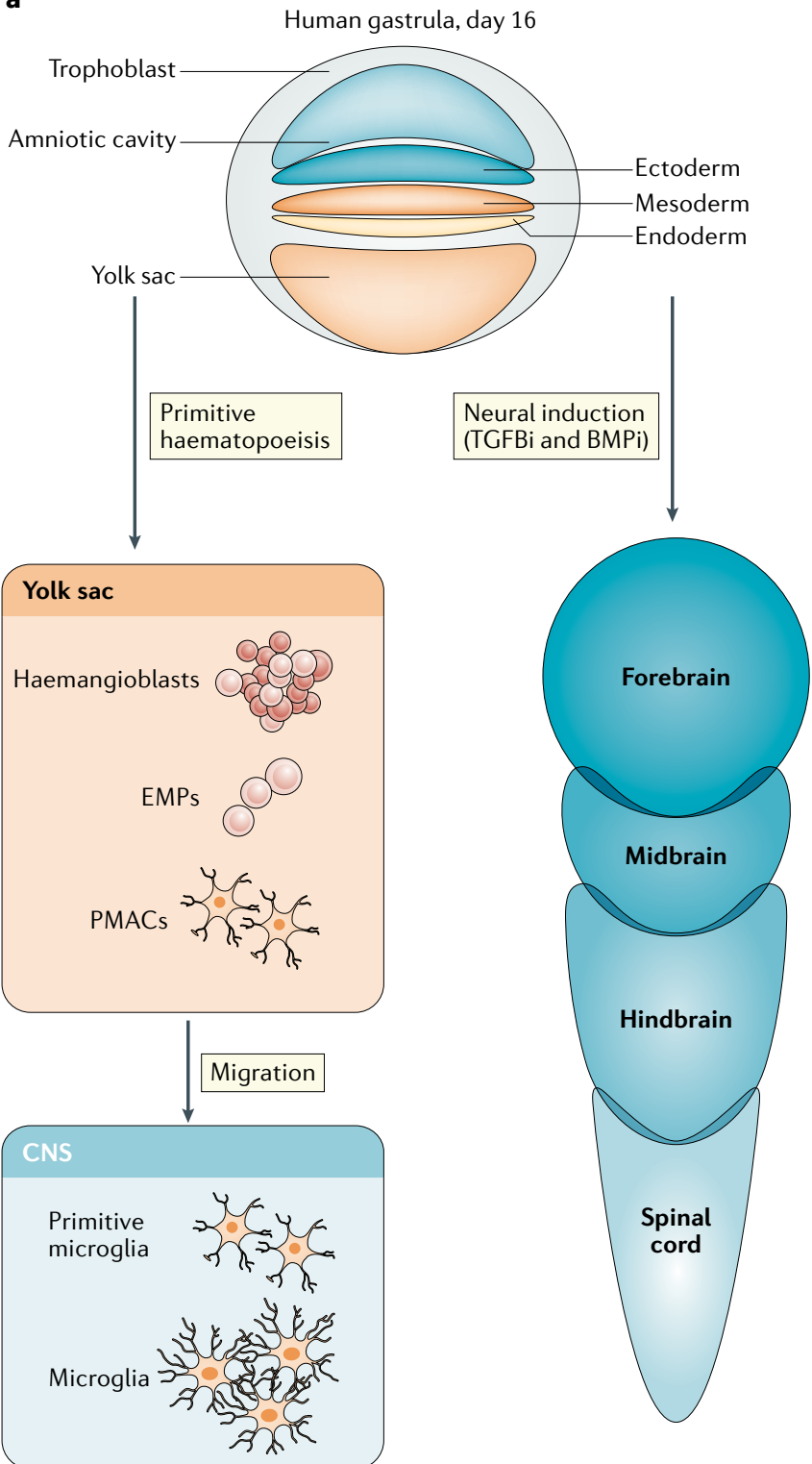

b
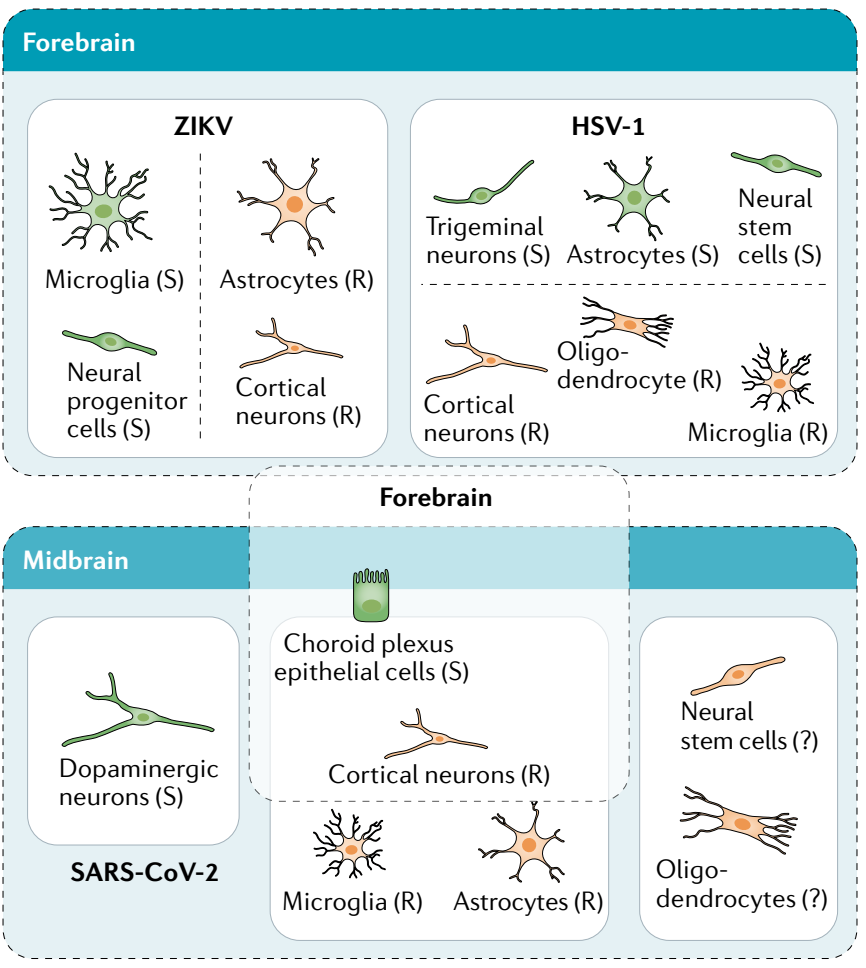

Hindbrain

DBR1 deficiency leading to hindbrain encephalitis Multiple neurotropic viruses

HSV-1

Norovirus

Influenza virus

Unknown cellular tropism in the hindbrain

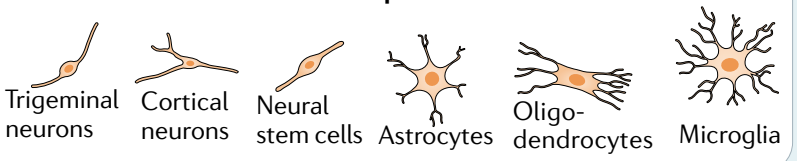

Fig. 1 | In vivo development guides in vitro differentiation principles to obtain disease-relevant cells. a | The major developmental pathways that govern in vitro differentiation strategies for microglia and neuroectodermal cell derivatives. b | Neurotropic viruses may target the central nervous system (CNS) in a region-specific or cell-specific manner. Indicated in the figure are examples of viruses that specifically target the forebrain (namely Zika virus (ZIKV) and herpes simplex virus 1 (HSV-1)), midbrain (severe acute respiratory syndrome coronavirus 2 (SARS-CoV-2)) and hindbrain (multiple neurotropic viruses in the context of DBR1 deficiency). Cells known to be susceptible to viral infection are coloured green and annotated with '(S)'. Cells annotated with '(R)' are resistant to infection and '(?)' indicates that the susceptibility of the cell population is not clear. $\mathrm{BMPi}$, bone morphogenetic protein inhibition; EMP, erythromyeloid progenitor; PMAC, premacrophage; TGFBi, transforming growth factor- $\beta$ inhibition.

motor neurons. An alternative strategy involves the overexpression of lineage-specific transcription factors to coax hPSCs into neural lineages. Such direct lineage conversion paradigms have been used to generate both neurons and glia ${ }^{63,65,67,68}$ as well as NPCs for high-content chemical screens or whole-genome genetic screens ${ }^{69}$. The advantages of direct lineage conversion include the increased speed at which cells acquire terminal fates and, in some instances, reduced variability among cells. However, their specific identity is often unclear, and for several protocols the resulting neurons may adopt a 'generic' neuron phenotype that does not exist in vivo rather than a specific neuronal subtype $e^{67,68}$.

Modelling primitive haematopoiesis in vitro and the derivation of human microglia. In contrast to neurons, astrocytes and oligodendrocytes, which are all neuroectoderm derivatives, microglia are yolk sac-derived CNS-resident macrophages that arise during primitive haematopoiesis ${ }^{70}$. There are two key components that determine the functional and molecular differences among tissue-resident macrophage populations, 
a Starting material
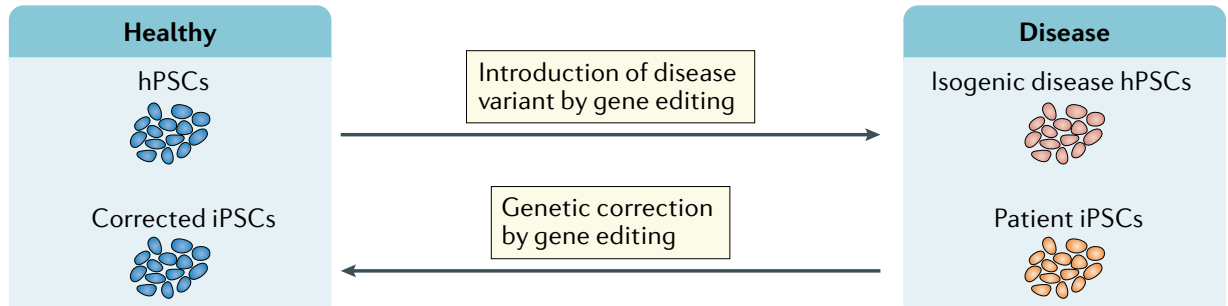

b

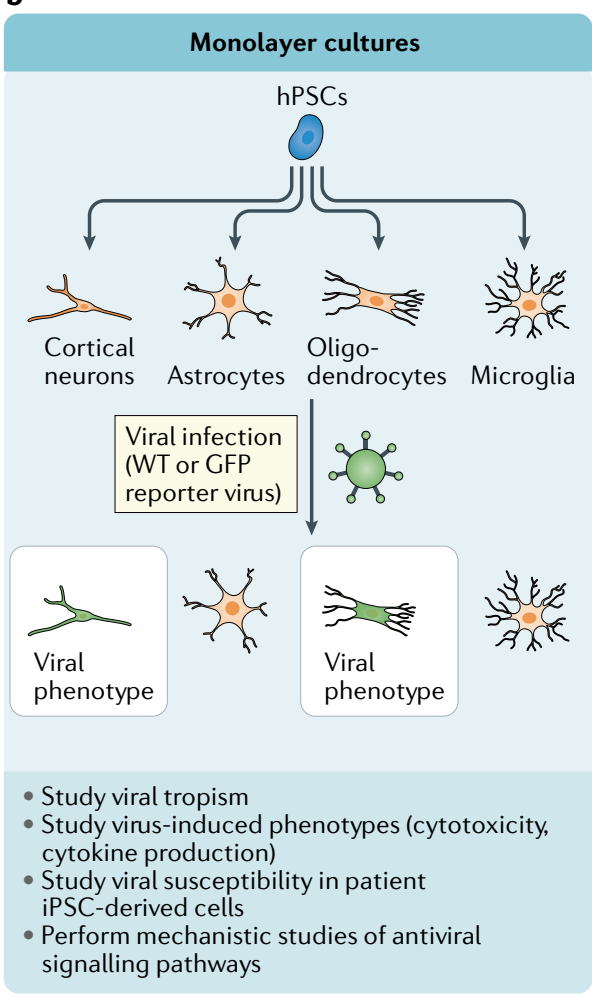

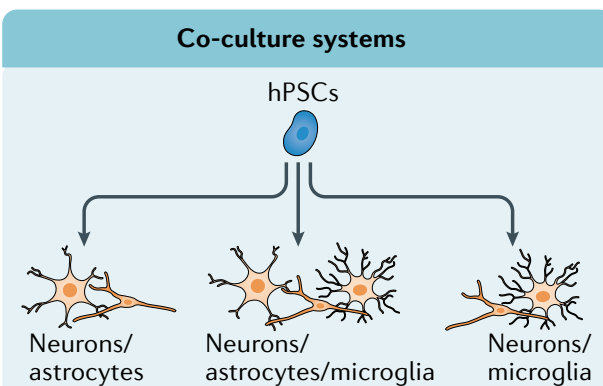
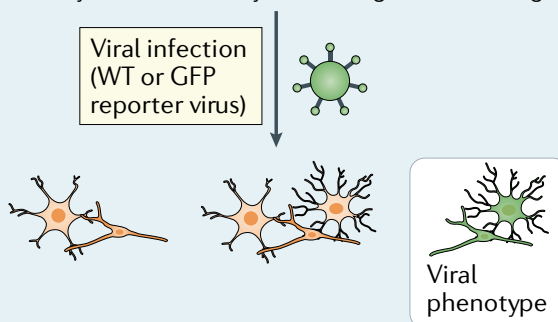

- Study cell-autonomous signalling pathways

- Co-culture systems are highly defined with

controllable cell ratios

- Still amenable to high-throughput screens

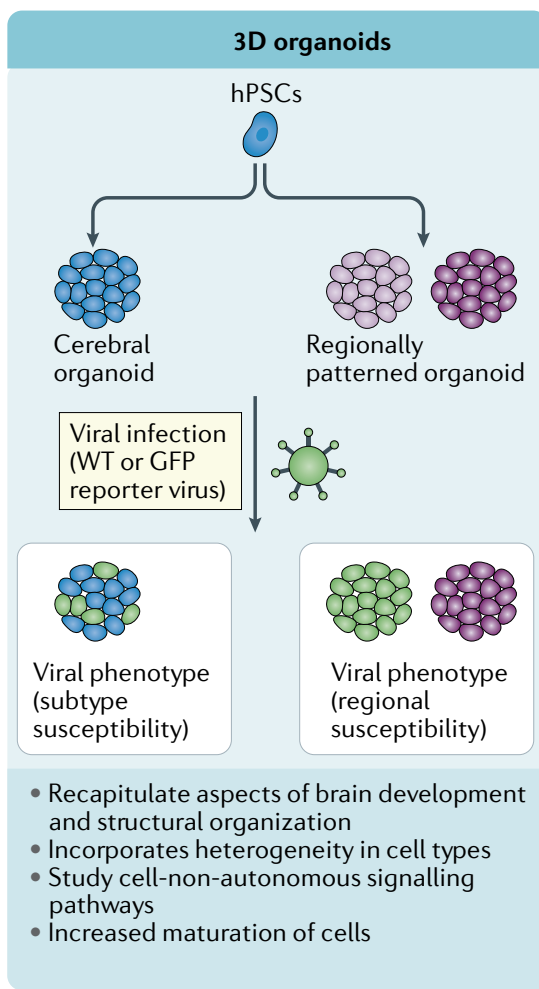

c

\section{Genome-wide screens to investigate host factors}

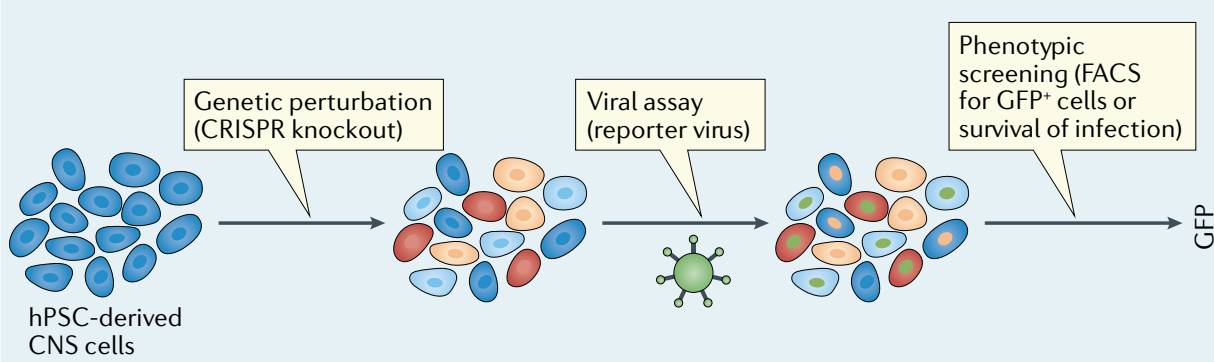

- Next-generation
sequencing
- Bioinformatics (enrichment
of sgRNAs and functional
classification of enriched
genes)

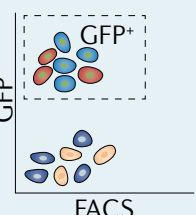

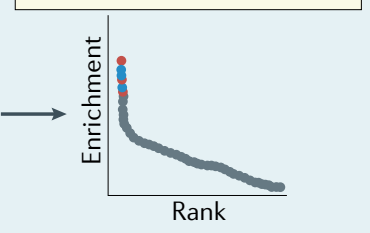

High-content chemical screens to identify antiviral compounds

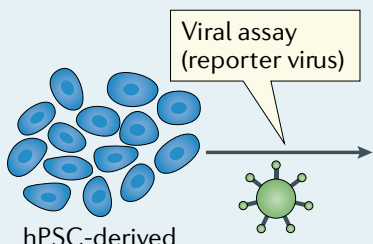

hPSC-derived CNS cells

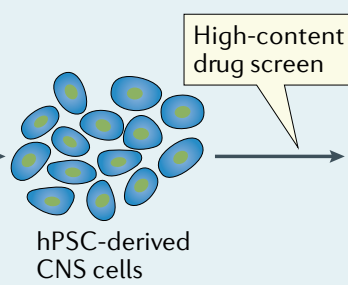

CNS cells
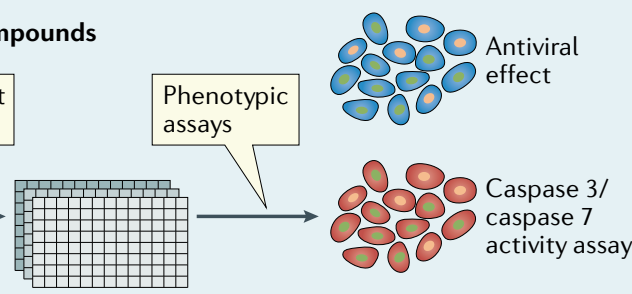

Cell viability assay

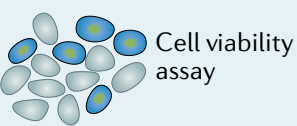


4 Fig. 2 | Applications of human pluripotent stem cells to study host-virus interactions. a | Human pluripotent stem cell (hPSC) technology in combination with genome editing tools allows the use of healthy hPSC lines versus those with genetically induced disease alleles, and the use of patient-derived induced pluripotent stem cell (iPSC) lines versus iPSC lines that have been genetically corrected. $\mathbf{b} \mid$ By differentiation of hPSCs into central nervous system (CNS) cells, host-virus interactions can be probed in monolayer cultures, co-culture systems or in more complex 3D organoids. Each of these differentiation paradigms can be applied to patient iPSCs or genetically engineered lines. $\mathbf{c}$ | Whole-genome genetic screens in hPSC-derived CNS cells can identify host factors that either promote viral replication or identify antiviral restriction factors in disease relevant cells that can lead to new therapeutic targets (top). An alternative approach to identify novel therapeutic candidates is the application of high-content chemical screens, which can yield antiviral compounds or compounds that ameliorate cytotoxicity (bottom). Cells (or nuclei) in green denote productive infection by the virus. FACS, fluorescence-activated cell sorting; sgRNA, single guide RNA; WT, wild type.

including microglia: namely, their ontogeny and their subsequent conditioning by tissue-specific niche factors ${ }^{71}$. Only recently have differentiation protocols been developed to recapitulate the developmental origin of microglia. By mimicking primitive streak specification (WNT and nodal $)^{72}$ followed by mesodermal differentiation (BMP4 and FGF2), haemogenic endothelial specification and haematopoietic transition (VEGF, IL-3, IL-6 and stem cell factor (SCF)), hPSCs convert sequentially into yolk sac macrophages and microglia-like cells ${ }^{73-77}$. In monocultures, these hPSC-derived microglia express several hallmark microglial markers, such as P2RY12, GPR34, TMEM119 and IBA1 (albeit at relatively low levels). However, they lack the required tissue-specific conditioning of the CNS, which can be overcome in part via co-culture with hPSC-derived neurons or astrocytes, incorporation into 3D cerebral organoids or transplantation into the mouse $\mathrm{CNS}^{78-82}$. While tissue-specific conditioning seems to play an important role in microglial differentiation, the specific factors involved in this process remain to be determined.

Increasing complexity by generating hPSC-derived cerebral organoids. There has been rapid progress in the development of hPSC-derived 3D cultures, such as cerebral spheroids and organoids. Early methods were based on promoting self-organization ${ }^{83-85}$, resulting in complex neuroepithelial structures, including cortical-like tissues ${ }^{85}$. Cerebral organoids can model features of early development, including NPC proliferation and differentiation and the establishment of cortical layers ${ }^{22}$. Furthermore, organoids provide a model where NPCs, neuronal cells and glial cells interact and develop in parallel. In addition to 'unguided' cerebral organoids ${ }^{22}$, region-specific forebrain, midbrain or hindbrain organoids can be obtained by adding specific patterning factors ${ }^{86,87}$. Alternative strategies include the introduction of a polarized patterning centre, resulting in multiple forebrain regions ${ }^{88}$ within the same organoid, or the fusion of independently patterned organoids to study the interaction of distinct brain regions. Finally, cells from different lineages, such as microglia or endothelial cells, can be introduced into cerebral organoids to obtain a closer representation of the CNS milieu ${ }^{82}$. While some of those lineages can develop spontaneously in unguided protocols ${ }^{89}$, it will be important to develop culture models that incorporate those cell types in a defined and highly reproducible manner.

Tailoring hPSC-based models for neuroimmunology. When hPSC-based disease models are used to study host-virus interactions in the CNS, there are several aspects to consider (FIC. 2). If the virus of interest affects a specific neurodevelopmental stage, an hPSC-based model must recapitulate that specific timing of development and include, for example, NPCs, in either 2D or $3 \mathrm{D}$ organoid cultures. For modelling region-specific disease, cells should be directed towards the respective CNS region. If a specific subpopulation of neurons or glial cells is affected, for example, cortical neurons, dopaminergic neurons, astrocytes or microglia, appropriate cell type-specific protocols should be used. Finally, for the study of cell-non-autonomous mechanisms, co-culture models or 3D cerebral organoids are required to examine cell-cell interactions. Remaining technical challenges include the derivation of fully mature (adult or aged) cell types and the need to further reduce variability, particularly in 3D organoid models.

\section{Limitations of hPSC-based disease modelling.} Considerable progress has been made in using hPSC technology and directed differentiation methods to derive disease-relevant cell types in a reproducible manner. Despite this, technical challenges remain that prevent hPSC-based models from reaching their full potential. A key limitation is that the current models are more suited towards developmental disorders rather than those occurring in the adult or aged CNS. Therefore, hPSC-based models are more suitable for studying viruses that target the developing brain, such as ZIKV, rather than infectious diseases that occur at later stages of life. Attempts to age hPSC-derived cells in vitro have been pursued ${ }^{90}$; however, those strategies may not replicate ageing in a physiological manner. Organoid models allow extended in vitro periods ranging from months to years of culture, which can increase the extent of in vitro maturation and ageing, and the first studies comparing hPSC-derived organoid models with their in vivo counterparts have proven hopeful ${ }^{11}$. However, such long-term culture is laborious and costly for routine use, and may be difficult to replicate more broadly. While the development of 3D organoid models has provided further opportunities to study development and disease in the CNS, these more complex models often show more variability than their $2 \mathrm{D}$ counterparts. It will be essential to further optimize these differentiation paradigms to make them more robust and carefully characterize their cellular composition and development over time.

Finally, while the available hPSC-based models are ideally suited to study the tropism of neurotropic viruses and potential virus-induced disease, they fail to address how viruses enter the $\mathrm{CNS}$ as they lack a $\mathrm{BBB}$ or diffusion kinetics. However, advances have been made by developing hPSC-derived BBB models using microfluidic technology ${ }^{92}$, hPSC-derived organoids with a functional vascular system ${ }^{93}$ and most recently choroid plexus organoids, which established that infection with SARS-CoV-2 disrupts the blood-cerebrospinal fluid barrier ${ }^{94}$. 
Table 1 | Human stem cell models for CNS viral infections

\begin{tabular}{|c|c|c|c|c|c|c|}
\hline Pathogen & Virus family & $\begin{array}{l}\text { Viral } \\
\text { genome }\end{array}$ & Target cell & hPSC-based model & Phenotype & Refs \\
\hline Zika virus & Flaviviridae & ssRNA & $\begin{array}{l}\text { NPCs, microglia, } \\
\text { macrophages }\end{array}$ & $\begin{array}{l}\text { Monocultures (NPCs), } \\
\text { co-cultures (neurons } \\
\text { and microglia), } \\
\text { cortical organoids }\end{array}$ & $\begin{array}{l}\text { Impaired organoid growth, premature } \\
\text { differentiation and apoptosis of NPCs, viral } \\
\text { replication in macrophages and microglia }\end{array}$ & $\begin{array}{r}32,33,86, \\
95,97-102, \\
107,109, \\
132-134\end{array}$ \\
\hline Dengue virus & Flaviviridae & ssRNA & NPC, macrophages & $\begin{array}{l}\text { Monocultures } \\
\text { (NPCs, neurons) }\end{array}$ & $\begin{array}{l}\text { Proinflammatory cytokine production in } \\
\text { NPCs, decreased migration of macrophages }\end{array}$ & 132,133 \\
\hline $\begin{array}{l}\text { Herpes simplex } \\
\text { virus } 1\end{array}$ & Herpesviridae & dsDNA & $\begin{array}{l}\text { NPCs, cortical } \\
\text { neurons, sensory } \\
\text { neurons, astrocytes, } \\
\text { oligodendrocytes }\end{array}$ & $\begin{array}{l}\text { Monocultures } \\
\text { (NPCs, astrocytes, } \\
\text { neurons, } \\
\text { oligodendrocytes) }\end{array}$ & $\begin{array}{l}\text { Cortical neurons and oligodendrocytes } \\
\text { derived from TLR3-and UNC93B-deficient } \\
\text { hPSCs show increased susceptibility to } \\
\text { infection, as is also the case for hPSC-derived } \\
\text { cortical neurons deficient in SNORA31, } \\
\text { while wild-type trigeminal neurons lack } \\
\text { cell-intrinsic viral resistance }\end{array}$ & $\begin{array}{r}30,31,35, \\
113,134-137\end{array}$ \\
\hline $\begin{array}{l}\text { Human } \\
\text { cytomegalovirus }\end{array}$ & Herpesviridae & dsDNA & NPCs & $\begin{array}{l}\text { Monocultures } \\
\text { (NPCs) }\end{array}$ & $\begin{array}{l}\text { Cytotoxicity in NPCs, impaired organoid } \\
\text { growth and structural development }\end{array}$ & $134,138-141$ \\
\hline Rabies lyssavirus & Rhabdoviridae & ssRNA & Neurons & $\begin{array}{l}\text { Monocultures } \\
\text { (neurons) }\end{array}$ & $\begin{array}{l}\text { Proinflammatory cytokine production and } \\
\text { axonal transmission of virus }\end{array}$ & 142 \\
\hline HIV & Retroviridae & ssRNA & Microglia & $\begin{array}{l}\text { Tricultures } \\
\text { (neurons, astrocytes } \\
\text { and microglia) }\end{array}$ & $\begin{array}{l}\text { Microglia are susceptible to HIV infection } \\
\text { and show inflammatory transcriptional } \\
\text { profiles and impaired phagocytosis }\end{array}$ & 46 \\
\hline
\end{tabular}

CNS, central nervous system; dsDNA, double-stranded DNA; hPSC, human pluripotent stem cell; NPC, neural progenitor cell; ssRNA, single-stranded RNA;

TLR3, Toll-like receptor 3 .

Using hPSCs to study CNS infections

Viral infections of the CNS. Viral encephalitis is most commonly caused by herpesviruses, arboviruses and enteroviruses ${ }^{38}$ and often triggers severe neurological damage. The factors that determine susceptibility to viral encephalitis remain poorly understood. While age-related decline in innate and adaptive immunity can cause increased susceptibility in older individuals (age $>60$ years), inborn errors of immunity that impair cell-intrinsic antiviral mechanisms can predispose children to viral encephalitis. Furthermore, some viruses induce disease at specific developmental stages, such as in the case of ZIKV infection, a disease process that has been studied in particular detail using hPSC-based models (see TABLE 1 for an overview of hPSC-based disease modelling of host-virus interactions).

hPSC-based models for ZIKV infection. ZIKV is an arbovirus belonging to the genus Flavivirus discovered in Uganda in 1947. Transfer of ZIKV to humans via Aedes aegypti mosquitoes was first reported in 2007. Infection with ZIKV in adults typically leads to mild symptoms. However, concurrent neurological manifestations, including microcephaly in newborns and GuillainBarré syndrome in adults, have caused major concerns. hPSC technology has played a key role in determining the tropism of ZIKV for different CNS cell types and in addressing the molecular mechanisms underlying the devastating microcephaly. Two early landmark studies using 2D monolayer cultures of hPSC-derived NPCs and neurons ${ }^{95}$ or $3 \mathrm{D}$ neurospheres ${ }^{32,33}$, respectively, showed that ZIKV preferentially infected NPCs, causing cell cycle dysregulation and cell death ${ }^{32,33,95}$. As a control, neurospheres were exposed to dengue virus serotype 2, a flavivirus with genetic similarities to ZIKV, which did not trigger cell death or abrogate NPC growth ${ }^{33}$. Qian and colleagues examined the neurotropism of ZIKV in more detail using a platform to establish forebrain-, midbrainand hypothalamic-specific cerebral organoids ${ }^{86}$. By deriving well-defined forebrain organoids which mimic the multilayer organization seen in human CNS development, they tested ZIKV infection at multiple developmental stages in vitro. While NPCs could be infected at all stages of in vitro differentiation, only early-stage forebrain organoids, containing a high proportion of SOX2 $2^{+}$ NPCs as opposed to TBR2 ${ }^{+}$progenitor cells or CTIP2 ${ }^{+}$ neurons, were vulnerable to ZIKV infection, resulting in cell death and decreased proliferation. These findings indicate a narrow temporal window during which the fetus is at risk. An additional study using both mouse pluripotent stem cell-based and hPSC-based models, described similar results and reported ZIKV-induced intrauterine growth retardation and microcephaly in mice (however, this was mouse strain dependent). In hPSC-derived cerebral organoids, the study authors observed ZIKV-mediated cell death due to apoptosis and autophagy, and proposed that increased susceptibility in NPCs could be attributed to their higher expression of AXL, a candidate receptor for ZIKV infection $^{32,96}$. Nevertheless, genetic ablation of AXL in hPSC-derived NPCs and cerebral organoids did not protect from ZIKV infection or ZIKV-induced cell death ${ }^{97}$. 
TYRO3, an alternative candidate viral receptor, was also highly expressed in both NPCs and cerebral organoids, and could play a role in ZIKV entry ${ }^{97}$. Alternatively, AXL and TYRO3 could serve as co-receptors in NPCs.

ZIKV disrupts NPC proliferation through distinct mechanisms. While multiple studies reported effects of ZIKV on $\mathrm{NPCs}^{32,33,86,95}$, the molecular mechanisms underlying this phenotype remained elusive. With use of a combination of mouse models and hPSC-derived forebrain organoids, NS2A, one of the seven non-structural ZIKV proteins, was shown to interact with and destabilize adherens junctions, resulting in aberrant radial glial organization ${ }^{98}$. Importantly, the interaction between ZIKV NS2A and adherens junction components impairs NPC proliferation and induces premature differentiation. An earlier study using fetal neural stem cells demonstrated inhibition of the AKT-mechanistic target of rapamycin signalling pathway by two other ZIKV non-structural proteins, NS4A and NS4B, leading to reduced neurogenesis and increased autophagy ${ }^{99}$. Additional evidence for ZIKV cytotoxicity in NPCs was presented via the heterodimeric protease of two ZIKV proteins, NS2B and NS3, interacting with the host protein septin 2 and leading to defects in cytokinesis and cell death ${ }^{100}$.

The role of non-neural cells in ZIKV infection. The role of glia during ZIKV infection has been addressed in hPSC-derived astrocytes and microglia-like cells ${ }^{101,102}$. Muffat and colleagues showed divergent innate immune responses in NPCs versus astrocytes or microglia. In line with previous studies, NPCs were highly susceptible to ZIKV infection, resulting in significant cell death ${ }^{102}$. By contrast, astrocytes and microglia did not show signs of cell death but rather served as reservoirs for productive viral replication ${ }^{101,102}$. The upregulation of candidate viral entry receptors on microglia (AXL, TYRO3 and TLR7) and proinflammatory markers (IL-6, IL- $1 \beta$ and CCR5) was ZIKV strain dependent ${ }^{101}$, potentially explaining disparate effects of African and Asian ZIKV strains on CNS phenotypes. Transfer of infected microglia in co-culture induced NPC infection and death ${ }^{101}$. These results led to the hypothesis that microglia may be a ZIKV vector enabling crossing of the placental-fetal barrier, as they originate in the yolk sac near the maternal vasculature before migrating into the developing CNS. This is in agreement with data showing ZIKV infecting and replicating in Hofbauer cells ${ }^{103}$, a type of human placental macrophage, and with a study in mice describing a very narrow time window when intrauterine ZIKV injection triggers fetal brain infection ${ }^{104}$ matching the exact time point when microglial progenitors enter the $\mathrm{CNS}^{104,105}$. Alternatively, as fetal macrophages as a whole are susceptible to ZIKV infection, peripheral blood macrophages could also serve as a Trojan horse for ZIKV infection into the developing brain by crossing the $\mathrm{BBB}^{42}$.

Chemical and genetic screens for ZIKV. hPSC models are ideally positioned for high-content chemical or genetic screens to identify therapeutic targets of ZIKV infection or other viral infections ${ }^{106}$. Whole-genome CRISPR-Cas screens can identify host factors involved in viral entry, replication, packaging and release, and are performed directly in disease-relevant hPSC-derived cell types. Li et al. used this strategy in hPSC-derived NPCs to identify molecular targets for potential therapeutic intervention ${ }^{107}$. They made several interesting observations; firstly, genes encoding putative ZIKV receptors (AXL and TYRO3) identified in previous studies in immortalized cell lines were not among the positive hits, highlighting the importance of using disease-relevant cells. Among the positive hits were genes involved in heparan sulfation, endocytosis, endoplasmic reticulum processing, Golgi apparatus function and interferon activity. These potentially relate to different stages of the viral cycle, namely viral entry (heparan sulfation, endocytosis), translation and replication of the viral genome (endoplasmic reticulum), egress from the host (Golgi apparatus) and host antiviral response (interferon activity). Hit validation was performed with use of single-gene knockouts and pharmacological interventions. A second whole-genome screen was based on NPCs derived via direct lineage conversion of hPSCs, and confirmed several hits and pathways involved (heparan sulfation, endocytosis, and Golgi apparatus function) ${ }^{69}$. Again, no evidence was found that either $A X L$ or TYRO3 plays a role in ZIKV infection in NPCs. Two other studies identified promising therapeutic candidates by performing high-content chemical screens of FDA-approved compounds ${ }^{108,109}$. Such drug-repurposing screens are designed to rapidly identify compounds of direct therapeutic relevance. The first study screened more than 6,000 compounds in hPSC-derived astrocytes and NPCs to either inhibit ZIKV infection or prevent subsequent cell death. Combining compounds from both groups improved response and was validated in hPSC-derived cerebral organoids. In the second study, we performed a screen of more than 1,000 FDAapproved drugs in hPSC-derived NPCs ${ }^{109}$. We validated two positive hits not only in hPSC-derived forebrain organoids in vitro but also in vivo in the adult mouse brain, and established that the key hit (hippeastrine hydrobromide) is not only prophylactic in preventing infection but can exert strong therapeutic anti-ZIKV activity several days after initial infection.

\section{Modelling viral latency and reactivation}

An area of virology where hPSC models could have a major impact is the study of viral latency and reactivation. After an initial productive or lytic phase of infection, some viruses undergo latency, whereby the full viral genome is retained in the host cell but with only few viral latency genes expressed and without production of infectious progeny. Latency can persist despite host immune responses and is reversible; thus, the virus can be reactivated, after which a new phase of productive infection occurs. While latency is mostly established in cells of the peripheral nervous system (PNS), it is important to CNS disease as reactivation of a latent infection may cause spread to the CNS $\mathrm{CS}^{35,110}$.

Herpesviruses can initiate both a lytic infection and a latent infection, with alphaherpesviruses, such as HSV-1 and varicella zoster virus, establishing latency specifically in the nervous system. The molecular mechanisms 

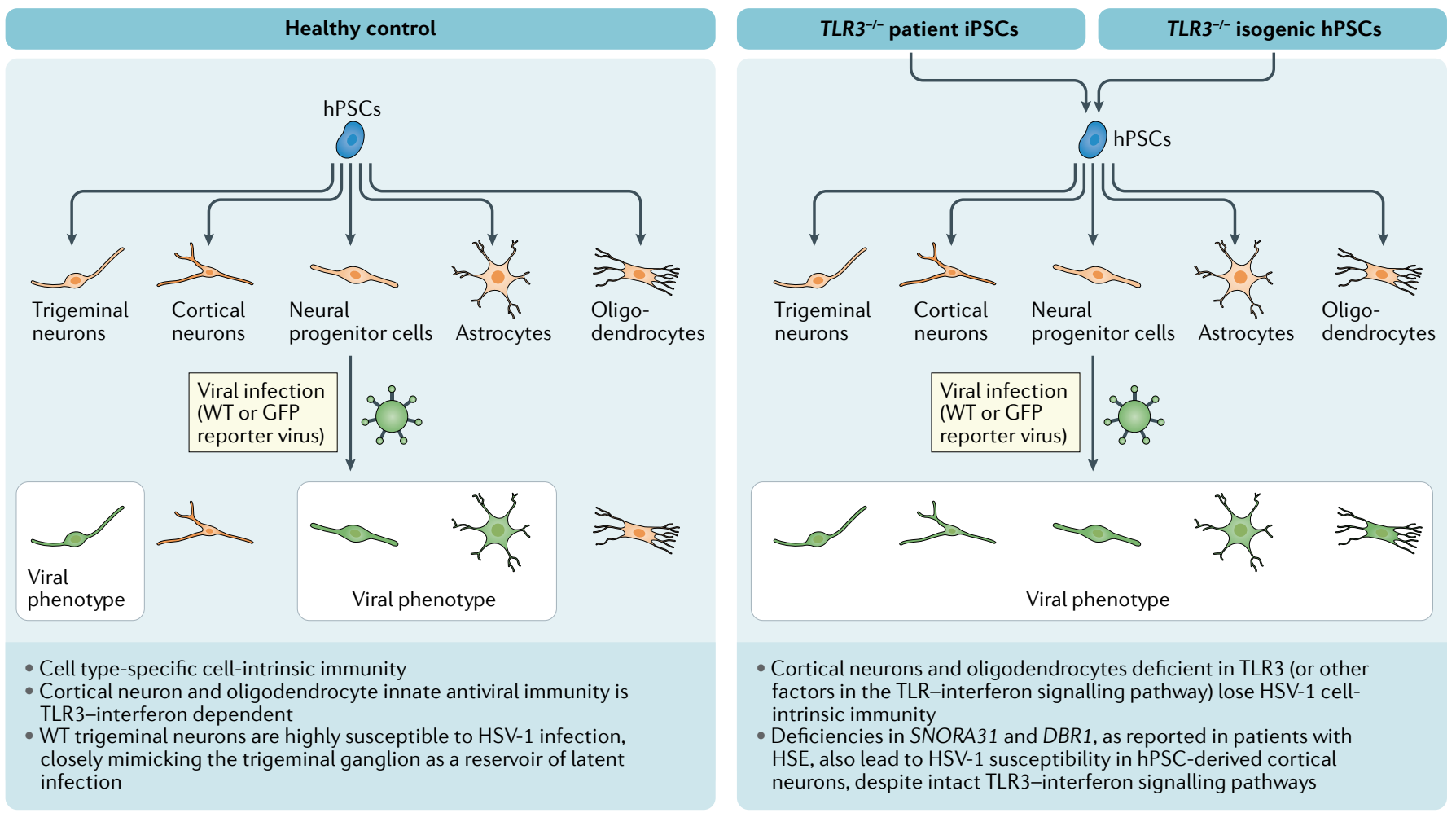

Fig. 3 | CNS immunity to HSV-1 is due to a cell-intrinsic, cell type-dependent TLR3-interferon response. Studies using human pluripotent stem cells (hPSCs) have dissected the differences in cell-intrinsic antiviral immunity to herpes simplex virus 1 (HSV-1) between various central nervous system (CNS) cell types. In healthy controls, hPSC-derived cortical neurons and oligodendrocytes show cell-intrinsic antiviral immunity to HSV-1, which is dependent on intact Toll-like receptor 3 (TLR3)-interferon signalling. This cell-intrinsic immunity is lost in patients who have deficiencies in the TLR3-interferon signalling pathway (due to genetic variants in TLR3, UNC93B or STAT1 for example) or in TLR3 $3^{-1-}$ isogenic control cells. hPSC-derived trigeminal neurons lack TLR3-interferon-dependent anti-HSV-1 immunity and show productive viral infection also in wild-type (WT) cells, closely mimicking the in vivo situation where trigeminal neurons are the reservoir for latent HSV-1 infection even in healthy individuals. Cells coloured green are productively infected by the virus. HSE, herpes simplex encephalitis.

of latency are poorly understood, with a lack of robust experimental models. While most in vitro models recapitulate only the lytic stage of infection, animal models are generally inefficient, expensive and time-consuming to study this phenomenon as latency is often established in very few cells and over protracted periods, making it difficult to acquire sufficient material to study viral gene expression. Furthermore, animal models lack the flexibility and scalability of hPSC-based models to enable whole-genome or chemical screens to determine host factors and therapeutic targets in studying viral latency.

With advances in hPSC-based disease modelling, studies have aimed to recapitulate viral latency and reactivation of HSV-1 and varicella zoster virus in vitro in human sensory neurons ${ }^{11-113}$. Some key criteria of viral latency could be established in vitro in hPSC-derived neurons: viral gene expression restricted to only a few key latency genes, viral genomes being episomal and non-replicative, and lack of infectious viral particle production. A key aspect in those studies was the maintenance of viral latency and the reactivation of latent infection. Reactivation of latent alphaherpesviruses can be debilitating and painful in the PNS, and can lead to life-threatening encephalitis on spread to the CNS. Many questions remain on how viral latency is established, on the selective vulnerability of distinct neuronal subtypes in the PNS, on whether all latent viral genomes are competent to reactivate and on whether a reactivated virus can re-establish latency ${ }^{110}$. Future studies using human stem cell models may address some of those key questions and facilitate the development of novel therapeutic strategies to prevent viral reactivation.

Modelling primary immunodeficiencies. To date, there are at least 430 inborn errors of immunity (also known as primary immunodeficiencies) ${ }^{114}$. Two known clinical entities where a genetic cause can enable CNS viral infection are herpes simplex encephalitis affecting the forebrain, caused by deficiencies in TLR3, UNC93B (also known as UNC93B1), TRAF3, TRIF (also known as TICAM1), TBK1 and IRF3 (REF. ${ }^{39}$, and viral encephalitis of the brainstem due to DBR1 deficiency, which can be caused by HSV-1, influenza virus or norovirus ${ }^{40}$.

Human iPSC technology has offered an ideal platform to study primary CNS immunodeficiencies. An early example of iPSC-based disease modelling for an inborn error of immunity affecting the human nervous system was the study of anti-HSV-1 immunity using multiple hPSC-derived CNS cells ${ }^{30}$ (FIG. 3). In this study, we reported that cell-intrinsic immunity for HSV-1 
infection in the CNS relies on an intact TLR3-interferon signalling pathway and is cell type dependent (see BOX 1 for details on cell-intrinsic antiviral signalling). Viral susceptibility and interferon production were studied in hPSC-derived, purified neural stem cells, cortical neurons, astrocytes and oligodendrocyte progenitor cells. Intriguingly, while cortical neurons and oligodendrocyte progenitor cells derived from healthy individuals could restrict viral replication of HSV-1, the corresponding cell types derived from TLR3- and UNC93B-deficient cells could not. This viral phenotype was explained by a lack of IFN- $\beta$ and IFN- $\lambda$ production in response to poly(I:C) (a TLR3 agonist) or HSV-1. By contrast, no such differences were seen in neural stem cells or astrocytes from either healthy or TLR3- and UNC93B-deficient cells. Furthermore, interferon production varied among neural stem cells, cortical neurons, astrocytes and oligodendrocyte progenitor cells following poly(I:C) stimulation. These experiments elegantly demonstrated that anti-HSV-1 immunity of the CNS is due to a cell-intrinsic, TLR3-interferon response in cortical neurons and oligodendrocyte progenitor cells.

A second study describes a directed differentiation paradigm to obtain human trigeminal neurons from hPSCs ${ }^{35}$. Trigeminal neurons, which are part of the PNS and developmentally originate from cranial placodes, play a key role in CNS infection as a site of entry and viral latency for HSV-1. Intriguingly, we reported distinct roles for trigeminal versus cortical neurons in HSV-1 host defence. While we confirmed a TLR3-interferon-dependent cell-intrinsic immunity to HSV-1 in cortical neurons, trigeminal neurons were highly susceptible to HSV-1 independently of whether they were derived from TLR3-deficient patients or from healthy individuals. This result mimics the viral life cycle of HSV-1, where the trigeminal ganglion becomes infected and a source for viral latency even in healthy individuals. Such hPSC-based platforms may provide

\section{Box 1 | Cell-intrinsic innate antiviral signalling}

A range of neurotropic viruses, including Zika virus, herpes simplex virus 1 (HSV-1), dengue virus, West Nile virus and varicella zoster virus, are capable of entering and infecting the central nervous system. To protect the host from viral infection, the innate immune system consists of four main classes of pattern recognition receptors (PRRs) that detect pathogen-associated molecular patterns ${ }^{143}$. The four classes of PRRs are Toll-like receptors (TLRs), retinoic acid-inducible gene 1 protein (RIG1) like receptors, nucleotide-binding oligomerization domain (NOD)-like receptors and cytosolic DNA sensors (such as cyclic GMP-AMP synthase). Signalling through these PRRs initiates antiviral immune pathways by activating the transcription factors nuclear factor- $\mathrm{kB}$ and interferon regulatory factors, leading to direct intrinsic antiviral immunity. Different subclasses of PRRs activate distinct innate immune signalling pathways; for example, detection of pathogen-associated molecular patterns by TLRs, such as HSV-1 by TLR3, leads to activation of myeloid differentiation primary response protein MYD88-dependent and Toll/IL-1 receptor domain-containing adaptor inducing interferon- $\beta$ (TRIF)-dependent signalling pathways and subsequent production of antiviral cytokines and type I interferons. The specificity of PRRs for a virus can be cell type specific. An example is that of human cortical neurons, which are dependent on TLR3-interferon signalling for their cell-intrinsic anti-HSV-1 immunity. By contrast, TLR3 is redundant for anti-HSV-1 immunity in haematopoietic cell types, explaining why patients with TLR3 deficiencies have infections limited to their central nervous system without dissemination to leukocytes or macrophages ${ }^{144}$. a powerful model to study molecular mechanisms of viral latency, as human primary trigeminal neurons are extremely difficult to isolate. Furthermore, trigeminal neurons from human cadavers have the caveat that they may have previously undergone HSV-1 infection and thus harbour latent viral infection.

In a recent study on the genetic susceptibility underlying herpes simplex encephalitis, hPSC-derived cortical neurons were used to establish a role for SNORA31, a small nucleolar RNA of the H/ACA class, as a CNS cell-intrinsic viral restriction factor ${ }^{31}$. Whole-exome sequencing identified five individuals susceptible to herpes simplex encephalitis carrying heterozygous rare variants in SNORA31. Using cortical neurons from both patient iPSCs and hPSCs with CRISP-Cas9-induced biallelic and monoallelic mutations, we established SNORA31 as essential for anti-HSV-1 immunity. Both patient and gene-edited lines for SNORA31 showed intact TLR3-interferon signalling and normal responses to TLR3 and IFN- $\alpha / \beta$ stimulation. However, transcriptomic analysis in hPSC-derived cortical neurons showed an impaired response to HSV-1 infection. Hence, while SNORA31 is a viral restriction factor for HSV-1, it does so independently of the known TLR3-interferon signalling pathways.

\section{Models for SARS-CoV-2 infection}

The coronavirus disease 2019 (COVID-19) pandemic and the broad tropism of SARS-CoV-2 for multiple organ systems and cell types highlights the importance of using disease-relevant models that can capture the cellular diversity of the human body, such as hPSC-based models. The importance of using human in vitro models is underscored by species barriers that can hinder translation of findings in primary cell lines to the clinic, even leading to false positive results that can waste precious time and resources that are of importance during a pandemic when a new infectious disease emerges ${ }^{115-117}$.

Although COVID-19 initially presented as a mostly acute respiratory disorder, it has become clear that that there are many extrapulmonary and even chronic manifestations ${ }^{118}$. These extrapulmonary manifestations include myocarditis, acute kidney injury, hepatocellular injury, gastrointestinal disease and increasing evidence for both PNS and CNS pathology ${ }^{118,119}$. There has been a rapid response from the stem cell field in engineering both 2D models and 3D organoids to study the tropism of SARS-CoV-2 in human gut enterocytes ${ }^{120}$ and intestinal organoids ${ }^{121,122}$, cardiomyocytes ${ }^{123-125}$, kidney organoids ${ }^{126}$, liver organoids ${ }^{124}$, pancreatic endocrine cells ${ }^{124}$ and, of course, lung organoids ${ }^{127}$.

In an early, modular hPSC-based platform to study SARS-CoV-2 tropism, we identified hPSC-derived dopaminergic neurons to be susceptible to SARS-CoV-2 infection, while hPSC-derived cortical neurons and microglia are resistant ${ }^{124}$. A second study that used both hPSC-derived monolayer cultures (neurons, astrocytes and microglia) and region-specific organoids (cortical, hippocampal, hypothalamic, midbrain and choroid plexus organoids) found that in all these human model systems, only choroid plexus organoids showed significant SARS-CoV-2 infection and replication (10-20\%), leading 
to inflammatory cellular responses and cell death ${ }^{128}$. These findings were corroborated by Pellegrini et al., who used hPSC-derived cortical organoids and choroid plexus organoids to study the tropism of SARS-CoV-2, and similarly found that while cortical neurons and astrocytes are relatively resistant to infection, there was productive infection in choroid plexus organoids ${ }^{94}$. These two studies indicate that the choroid plexus could potentially serve as an entry site for SARS-CoV-2 into the CNS, as productive infection in choroid plexus epithelial cells, due to virus circulating in nearby capillaries, could lead to viral spread into the cerebrospinal fluid.

However, conflicting data report a direct neurotropism of SARS-CoV-2 with use of hPSC-derived organoids, a mouse model overexpressing human ACE2, and COVID-19 patient brain autopsy material. The study authors present evidence for the neuroinvasive capacity of SARS-CoV-2 in neural stem cells and cortical neurons ${ }^{129}$, findings in direct contrast to those of previous reports. While the brain autopsy reports showed evidence of neuroinvasion by staining for anti-SARS-CoV-2 spike protein ${ }^{129}$, there was a striking lack of immune cell infiltration around infected sites. Furthermore, the association of SARS-CoV-2 infection in this study with sites of ischaemic infarcts that the patients had experienced may point to an alternative route for viral invasion into the CNS.

It was perhaps thought that the extrapulmonary manifestations would occur mostly in patients with severe COVID-19. This, however, does not seem to be the case, as is illustrated by the occurrence of myocarditis in relatively young and often otherwise asymptomatic patients ${ }^{130}$. Whether this is also true for the neurological manifestations of COVID-19 remains to be seen, as the true incidence is unknown. This is further complicated by the fact that neurological damage may not become apparent until much later, leading to postinfectious disease such as postencephalitic parkinsonism, which was described following the 1918 influenza epidemic ${ }^{44,45}$. There are still many open questions on how SARS-CoV-2 can affect the human nervous system, such as which subpopulations of neurons and non-neuronal cells are susceptible to SARS-CoV-2 infection, which clinical phenotypes are a direct result of infection in the CNS versus secondary to SARS-CoV-2mediated vascular damage or cytokine release, and whether CNS involvement could lead to long-term neurological damage or increased susceptibility to neurodegeneration. Studies with carefully characterized hPSC-derived CNS cell types are needed to resolve questions about the precise tropism of SARS-CoV-2 in the brain and to establish model systems that allow the study of SARS-CoV-2-mediated long-term effects.

\section{Conclusions}

To date, hPSC-based models have successfully been used to study viral infections of the CNS in NPCs, neurons, astrocytes, oligodendrocytes and microglia. Additionally, differentiation paradigms exist to derive cells of the PNS, such as trigeminal neurons, that play a role in viral latency and CNS entry. More broadly, hPSC technology has enabled neuroimmunologists to study both sporadic viral infections and primary CNS immunodeficiencies in disease-relevant human cells.

While 3D organoid models have shown considerable promise for the study of host-virus interactions, further progress is required to achieve models that more closely represent their in vivo equivalent and show reduced variability. Strategies towards this goal include the use of specialized spinning bioreactors ${ }^{86}$ or the introduction of signalling centres into organoids to ensure spatial topography ${ }^{88}$. The fusion of multiple organoids into a single assembloid could represent a model to recreate the physiological path of infection for viruses such as HSV-1. Other ways to increase complexity include the addition of multiple cell types in 2D co-culture systems or by use of microfluidic devices. Most CNS models do not include several non-neural cell types, such as endothelial cells, pericytes or microglia. While such lineages can develop spontaneously in unguided protocols ${ }^{89}$, it will be important to develop culture models that incorporate those cell types in a defined manner.

The combination of hPSC technology with genetic or chemical screens is a particularly attractive strategy to identify genetic host factors that drive viral susceptibility and to nominate candidate therapeutic targets for drug design or clinical treatment. Future screens may harness the potential of automated imaging technologies to apply such screens in more complex co-culture systems or $3 \mathrm{D}$ organoid models. With the incorporation of multiple CNS cell types in a single model, it will be intriguing to assess cell type-specific responses, and cell-cell interactions following a viral stimulus. One method to detect heterogeneity in pathogen responses is single-cell RNA sequencing (scRNA-seq). Studies using scRNA-seq should facilitate the dissection of cell type-specific innate immune responses (for example, neuronal versus microglial responses) and identify differences in cellular states within a population (for example, infected cells versus bystander cells and inflammatory states). Furthermore, scRNA-seq may offer unique insights into activation states after infection and help to unravel the mechanisms underlying long-term neurological deficits following viral encephalitis. By combining CRISPR-Cas-based gene editing with scRNA-seq (through a technique known as Perturb-seq), it is now possible to assay single-cell transcriptional profiles linked to a specific genotype and phenotype within a single experiment ${ }^{131}$. Such techniques could probe genetic regulators of an antiviral response in human disease-relevant cells, while simultaneously obtaining transcriptional data on the gene regulatory pathways involved during immune activation. This may lead to the rapid identification of important host factors that regulate antiviral immunity and the corresponding regulatory pathways involved.

Finally, in a similar way that the stem cell field reacted to the ZIKV health crisis, we currently have an opportunity to harness the power of hPSC-based models to address the COVID-19 pandemic. There are many open questions that hPSC-based models can help to address, such as the exact neurotropism of SARS-CoV-2, the molecular mechanisms underlying a differential viral 
susceptibility (for instance, expression of specific viral co-receptors by dopaminergic neurons versus cortical neurons), whether SARS-CoV-2 infection may lead to long-term neurological damage and the identification of therapeutic targets to treat neuroinflammation in patients with COVID-19. The first studies performing high-content chemical screens to identify therapeutic compounds that may alleviate SARS-CoV-2-induced disease using human cells from hPSC-derived or primary organoids have been published ${ }^{122,127}$, and it will be important to see whether hits identified in these studies can be translated to a clinical setting.
We have described several remaining hurdles to be overcome for hPSC-based models to reach their full potential in neuroimmunology. However, hPSC-based models have already proven to be invaluable tools for immunologists in a spectacularly short time, both in the case of sporadic viral infections of the CNS and for inborn errors of immunity leading to viral encephalitis. Human stem cell technology will provide an essential addition to the scientific toolbox of neuroimmunologists and help to translate findings to patients in need.

Published online 4 January 2021
1. Thomson, J. A. Embryonic stem cell lines derived from human blastocysts. Science 282, 1145-1147 (1998) This study describes the first example of deriving human embryonic stem cell lines from blastocysts.

2. Takahashi, K. et al. Induction of pluripotent stem cells from adult human fibroblasts by defined factors. Cell 131, 861-872 (2007).

This is first example of cellular reprogramming leading to the generation of human iPSCs from terminally differentiated, somatic cells.

3. Nicholas, C. R. et al. Functional maturation of hPSC-derived forebrain interneurons requires an extended timeline and mimics human neural development. Cell Stem Cell 12, 573-586 (2013).

4. Gafni, O. et al. Derivation of novel human ground state naive pluripotent stem cells. Nature $\mathbf{5 0 4}$ 282-286 (2013)

5. Deglincerti, A. et al. Self-organization of the in vitro attached human embryo. Nature 533, 251-254 (2016).

6. Martyn, I., Kanno, T. Y., Ruzo, A., Siggia, E. D. $\delta$ Brivanlou, A. H. Self-organization of a human organizer by combined Wnt and Nodal signalling. Nature 558, 132-135 (2018)

7. Karzbrun, E., Kshirsagar, A., Cohen, S. R., Hanna, J. H. \& Reiner, O. Human brain organoids on a chip reveal the physics of folding. Nat. Phys. 14, 515-522 (2018).

8. Dimos, J. T. et al. Induced pluripotent stem cells generated from patients with ALS can be differentiated into motor neurons. Science 321 1218-1221 (2008)

This study provides an early example of iPSC-based disease modelling of neurological disease using patient-derived cells.

9. Israel, M. A. et al. Probing sporadic and familial Alzheimer's disease using induced pluripotent stem cells. Nature 482, 216-220 (2012).

10. Soldner, F. et al. Parkinson-associated risk variant in distal enhancer of $\alpha$-synuclein modulates target gen expression. Nature 533, 95-99 (2016).

11. Paquet, D. et al. Efficient introduction of specific homozygous and heterozygous mutations using CRISPR/Cas9. Nature 533, 125-129 (2016).

12. Kriks, S. et al. Dopamine neurons derived from human ES cells efficiently engraft in animal models of Parkinson's disease. Nature 480, 547-551 (2011)

13. Shiba, Y. et al. Human ES-cell-derived cardiomyocytes electrically couple and suppress arrhythmias in injured hearts. Nature 489, 322-325 (2012).

14. Ma, L. et al. Human embryonic stem cell-derived GABA neurons correct locomotion deficits in quinolinic acid-lesioned mice. Cell Stem Cell 10, 455-464 (2012).

15. Wang, S. et al. Human iPSC-derived oligodendrocyte progenitor cells can myelinate and rescue a mouse model of congenital hypomyelination. Cell Stem Cell 12, 252-264 (2013)

16. Gornalusse, G. G. et al. HLA-E-expressing pluripotent stem cells escape allogeneic responses and lysis by NK cells. Nat. Biotechnol. 35, 765-772 (2017).

17. Kikuchi, T. et al. Human iPS cell-derived dopaminergic neurons function in a primate Parkinson's disease model. Nature 548, 592-596 (2017)

18. Deuse, T. et al. Hypoimmunogenic derivatives of induced pluripotent stem cells evade immune rejection in fully immunocompetent allogeneic recipients. Nat. Biotechnol. 37, 252-258 (2019).

19. Mestas, J. \& Hughes, C. C. W. Of mice and not men differences between mouse and human immunology. J. Immunol. 172, 2731-2738 (2004).
20. Sinmaz, N., Nguyen, T., Tea, F., Dale, R. C. \& Brilot, F. Mapping autoantigen epitopes: molecular insights into autoantibody-associated disorders of the nervous system. J. Neuroinflammation 13, 219 (2016).

21. Smith, A. M. $\&$ Dragunow, M. The human side of microglia. Trends Neurosci. 37, 125-135 (2014).

22. Lancaster, M. A. et al. Cerebral organoids model human brain development and microcephaly. Nature 501, 373-379 (2014).

This is one of the early reports of hPSC-derived cerebral organoids and their application for the disease modelling of microcephaly.

23. Ebert, A. D. et al. Induced pluripotent stem cells from a spinal muscular atrophy patient. Nature $\mathbf{4 5 7}$, 277-280 (2009)

24. Zeltner, N. et al. Capturing the biology of disease severity in a PSC-based model of familial dysautonomia. Nat. Med. 22, 1421-1427 (2016).

25. Soldner, F. et al. Generation of isogenic pluripotent stem cells differing exclusively at two early onset Parkinson point mutations. Cell 146, 318-331 (2011).

26. Harschnitz, O. Human stem cell-derived models: lessons for autoimmune diseases of the nervous system. Neuroscientist 25, 199-207 (2018).

27. Steinbeck, J. A et al. Functional connectivity under optogenetic control allows modeling of human neuromuscular disease. Cell Stem Cell 18, 134-143 (2015).

28. Harschnitz, O. \& van den Berg, L. H. Autoantibody pathogenicity in a multifocal motor neuropathy induced pluripotent stem cell-derived model. Ann. Neurol. 80, 71-88 (2016).

29. Clark, A. J., Kaller, M. S., Galino, J., Willison, H. J., Rinaldi, S. \& Bennett, D. L. H. Co-cultures with stem cell-derived human sensory neurons reveal regulators of peripheral myelination. Brain 140, 898-913 (2017).

30. Lafaille, F. G. et al. Impaired intrinsic immunity to HSV- 1 in human iPSC-derived TLR3-deficient CNS cells. Nature 491, 769-773 (2012).

31. Lafaille, F. G. et al. Human SNORA31 variations impair cortical neuron-intrinsic immunity to HSV-1 and underlie herpes simplex encephalitis. Nat. Med. 25, 1873-1884 (2019).

Lafaille et al. (2012) and Lafaille et al. (2019) describe examples of hPSC-based disease modelling for primary immunodeficiencies that lead to a loss of cell-intrinsic immunity in cortical neurons to HSV-1 infection

32. Cugola, F. R. et al. The Brazilian Zika virus strain causes birth defects in experimental models. Nature 534, 267-271 (2016).

33. Garcez, P. P. et al. Zika virus impairs growth in human neurospheres and brain organoids. Science 352 , 816-818 (2016)

Cugola et al. (2016) and Garcez et al. (2016) are early examples of cerebral organoids being used to study the tropism and pathogenesis of ZIKV, identifying NPCs as a susceptible cell type

34. Delhaye, S. et al. Neurons produce type I interferon during viral encephalitis. Proc. Natl Acad. Sci. USA 103, 7835-7840 (2006)

35. Zimmer, B. et al. Human iPSC-derived trigeminal neurons lack constitutive TLR3-dependent immunity that protects cortical neurons from HSV-1 infection. Proc. Natl Acad. Sci. USA 115, E8775-E8782 (2018).

36. Omalu, B. I., Shakir, A. A., Wang, G., Lipkin, W. I. $\&$ Wiley, C. A. Fatal fulminant pan-meningopolioencephalitis due to West Nile virus. Brain Pathol. 13, 465-472 (2003).
37. Cho, H. et al. Differential innate immune response programs in neuronal subtypes determine susceptibility to infection in the brain by positive-stranded RNA viruses. Nat. Med. 19, 458-464 (2013)

38. Tyler, K. L. Acute viral encephalitis. N. Engl. J. Med. 379, 557-566 (2018)

39. Jouanguy, E. et al. Human inborn errors of immunity to herpes viruses. Curr. Opin. Immunol. 62, 106-122 (2020).

40. Zhang, S.-Y. et al. Inborn errors of RNA lariat metabolism in humans with brainstem viral infection. Cell 172, 952-965.e18 (2018).

41. Qian, X., Nguyen, H. N., Jacob, F., Song, H. \& Ming, G.-L. Using brain organoids to understand Zika virus-induced microcephaly. Development 144 952-957 (2017).

42. Lum, F-M. et al. Zika virus infects human fetal brain microglia and induces inflammation. Clin. Infect. Dis. 64, 914-920 (2017).

43. Di Liberto, G. et al. Neurons under T cell attack coordinate phagocyte-mediated synaptic stripping Cell 175, 458-471.e19 (2018).

44. Dale, R. C. et al. Encephalitis lethargica syndrome: 20 new cases and evidence of basal ganglia autoimmunity. Brain 127, 21-33 (2004).

45. Hoffman, L. A. \& Vilensky, J. A. Encephalitis lethargica: 100 years after the epidemic. Brain 140, 2246-2251 (2017)

46. Ryan, S. K. et al. Neuroinflammation and EIF2 signaling persist despite antiretroviral treatment in an hiPSC tri-culture model of HIV infection Stem Cell Rep. 14, 703-716 (2020).

47. Reinert, L. S. et al. Sensing of HSV-1 by the cCAS-STING pathway in microglia orchestrates antiviral defence in the CNS. Nat. Commun. 7, 13348 (2016).

48. Chhatbar, C. et al. Type I interferon receptor signaling of neurons and astrocytes regulates microglia activation during viral encephalitis. Cell Rep. 25, 118-129.e4 (2018).

49. Vasek, M. J. et al. A complement-microglial axis drives synapse loss during virus-induced memory impairment. Nature 534, 538-543 (2016).

50. Bialas, A. R. et al. Microglia-dependent synapse loss in type I interferon-mediated lupus. Nature 546, 539-543 (2017)

51. Garber, C. et al. T cells promote microglia-mediated synaptic elimination and cognitive dysfunction during recovery from neuropathogenic flaviviruses. Nat. Neurosci. 22, 1276-1288 (2019).

52. Blank, T. et al. Brain endothelial- and epithelial-specific interferon receptor chain 1 drives virus-induced sickness behavior and cognitive impairment. Immunity 44, 901-912 (2016)

53. Lee, S. H., Lumelsky, N., Studer, L., Auerbach, J. M \& McKay, R. D. Efficient generation of midbrain and hindbrain neurons from mouse embryonic stem cells. Nat. Biotechnol. 18, 675-679 (2000).

54. Chambers, S. M. et al. Highly efficient neural conversion of human ES and iPS cells by dual inhibition of SMAD signaling. Nat. Biotechnol. 27, 275-280 (2009).

55. Chambers, S. M. et al. Combined small-molecule inhibition accelerates developmental timing and converts human pluripotent stem cells into nociceptors. Nat. Biotechnol. 30, 715-720 (2012).

56. Liu, Y. et al. Medial ganglionic eminence-like cells derived from human embryonic stem cells correct learning and memory deficits. Nat. Biotechnol. 31 440-447 (2013).

57. Liu, Y. et al. Directed differentiation of forebrain GABA interneurons from human pluripotent stem cells. Nat. Protoc. 8, 1670-1679 (2013). 
58. Maroof, A. M. et al. Directed differentiation and functional maturation of cortical interneurons from human embryonic stem cells. Cell Stem Cell 12 559-572 (2013)

59. Du, Z.-W. et al. Generation and expansion of highly pure motor neuron progenitors from human pluripotent stem cells. Nat. Commun. 6, 6626 (2015)

60. Lu, J. et al. Generation of serotonin neurons from human pluripotent stem cells. Nat. Biotechnol. 34 89-94 (2016)

61. Krencik, R., Weick, J. P., Liu, Y., Zhang, Z.-J. \& Zhang, S.-C. Specification of transplantable astroglial subtypes from human pluripotent stem cells. Nat. Biotechnol. 29, 528-534 (2011).

62. Li, X. et al. Fast generation of functional subtype astrocytes from human pluripotent stem cells. Stem Cell Rep. 11, 998-1008 (2018).

63. Tchieu, J. et al. NFIA is a gliogenic switch enabling rapid derivation of functional human astrocytes from pluripotent stem cells. Nat. Biotechnol. 37, 267-275 (2019)

64. Bradley, R. A. et al. Regionally specified human pluripotent stem cell-derived astrocytes exhibit different molecular signatures and functional properties. Development 146, dev170910 (2019).

65. Yang, N. et al. Generation of oligodendroglial cells by direct lineage conversion. Nat. Biotechnol. $\mathbf{3 1}$ 434-439 (2013)

66. Tao, Y. \& Zhang, S.-C. Neural subtype specification from human pluripotent stem cells. Cell Stem Cell 19, 573-586 (2016)

67. Vierbuchen, T. et al. Direct conversion of fibroblasts to functional neurons by defined factors. Nature $\mathbf{4 6 3}$, 1035-1041 (2010)

68. Pang, Z. P. et al. Induction of human neuronal cells by defined transcription factors. Nature 476, 220-223 (2012)

69. Wells, M. F. et al. Genome-wide screens in accelerated human stem cell- derived neural progenitor cells identify Zika virus host factors and drivers of proliferation. Preprint at bioRxiv https://doi.org/ 10.1101/476440 (2018).

70. Thion, M. S., Ginhoux, F. \& Garel, S. Microglia and early brain development: an intimate journey. Science 362, 185-189 (2018).

71. Lee, C. Z. W., Kozaki, T. \& Ginhoux, F. Studying tissue macrophages in vitro: are iPSC-derived cells the answer? Nat Rev Immunol 18, 716-725 (2018).

72. Sturgeon, C. M., Ditadi, A., Awong, G., Kennedy, M. $\&$ Keller, G. Wnt signaling controls the specification of definitive and primitive hematopoiesis from human pluripotent stem cells. Nat. Biotechnol. 32, 554-561 (2014).

73. Muffat, J. et al. Efficient derivation of microglia-like cells from human pluripotent stem cells. Nat. Med. 22, 1358-1367 (2016)

74. Abud, E. M. et al. iPSC-derived human microglia-like cells to study neurological diseases. Neuron $\mathbf{9 4}$ 278-293.e9 (2017).

75. Pandya, $\mathrm{H}$. et al. Differentiation of human and murine induced pluripotent stem cells to microglia-like cells. Nat. Neurosci. 20, 753-759 (2017).

76. Douvaras, P. et al. Directed differentiation of human pluripotent stem cells to microglia. Stem Cell Rep. $\mathbf{8}$ 1516-1524 (2017)

77. Takata, K et al Induced-pluripotent-stem-cell-derived primitive macrophages provide a platform for modeling tissue-resident macrophage differentiation and function. Immunity 47, 183-198.e6 (2017).

78. Haenseler, W. et al. A highly efficient human pluripotent stem cell microglia model displays a neuronal-co-culture-specific expression profile and inflammatory response. Stem Cell Rep. 8, 1727-1742 (2017)

79. Hasselmann, J. et al. Development of a chimeric model to study and manipulate human microglia in vivo. Neuron 103, 1016-1033 e10 (2019).

80. Svoboda, D. S. et al. Human iPSC-derived microglia assume a primary microglia-like state after transplantation into the neonatal mouse brain Proc. Natl Acad. Sci. USA 116, 25293-25303 (2019).

81. Mancuso, R. et al. Stem-cell-derived human microglia transplanted in mouse brain to study human disease. Nat. Neurosci. 22, 2111-2116 (2019).

82. Schmunk, G. et al. Human microglia upregulate cytokine signatures and accelerate maturation of neural networks. Preprint at bioRxiv https://doi.org/ 10.1101/2020.03.24.006874 (2020).

83. Zhang, S.-C., Wernig, M., Duncan, I. D., Brüstle, O. \& Thomson, J. A. In vitro differentiation of transplantable neural precursors from human embryonic stem cells. Nat. Biotechnol. 19, 1129-1133 (2001).

84. Watanabe, K. et al. A ROCK inhibitor permits survival of dissociated human embryonic stem cells. Nat. Biotechnol. 25, 681-686 (2007).

85. Eiraku, M. et al. Self-organized formation of polarized cortical tissues from ESCs and its active manipulation by extrinsic signals. Cell Stem Cell 3, 519-532 (2008).

86. Qian, X. et al. Brain-region-specific organoids using mini-bioreactors for modeling ZIKV exposure. Cell 165, 1238-1254 (2016).

87. Jo, J. et al. Midbrain-like organoids from human pluripotent stem cells contain functional dopaminergic and neuromelanin-producing neurons. Cell Stem Cell 19, 248-257 (2016)

88. Cederquist, G. Y. et al. Specification of positional identity in forebrain organoids. Nat. Biotechnol. 37 , 436-444 (2019)

89. Ormel, P. R. et al. Microglia innately develop within cerebral organoids. Nat. Commun. 9, 4167 (2018).

90. Miller, J. D. et al. Human iPSC-based modeling of late-onset disease via progerin-induced aging. Cell Stem Cell 13, 691-705 (2013).

91. Cowan, C. S. et al. Cell types of the human retina and its organoids at single-cell resolution. Cell 182, 1623-1640.e34 (2020).

92. Vatine, G. D. et al. Human iPSC-derived blood-brain barrier chips enable disease modeling and personalized medicine applications. Cell Stem Cell 24, 995-1005.e6 (2019).

93. Cakir, B. et al. Engineering of human brain organoids with a functional vascular-like system. Nat. Methods 16, 1169-1175 (2019)

94. Pellegrini, L. et al. SARS-CoV-2 infects the brain choroid plexus and disrupts the blood-CSF-barrier in human brain organoids. Cell Stem Cell 27, 951-961.e5 (2020).

95. Tang, H. et al. Zika virus infects human cortical neural progenitors and attenuates their growth. Cell Stem Cell 18, 587-590 (2016)

96. Nowakowski, T. J. et al. Expression analysis highlights $\mathrm{AXL}$ as a candidate Zika virus entry receptor in neural stem cells. Cell Stem Cell 18, 591-596 (2016).

97. Wells, M. F. et al. Genetic ablation of AXL does not protect human neural progenitor cells and cerebral organoids from Zika virus infection. Cell Stem Cell 19, 703-708 (2016).

98. Yoon, K.-J. et al. Zika-virus-encoded NS2A disrupts mammalian cortical neurogenesis by degrading adherens junction proteins. Cell Stem Cell 21 349-358.e6 (2017).

99. Liang, Q. et al. Zika Virus NS4A and NS4B proteins deregulate Akt-mTOR signaling in human fetal neural stem cells to inhibit neurogenesis and induce autophagy. Cell Stem Cell 19, 663-671 (2016).

100. Li, H. et al. Zika virus protease cleavage of host protein Septin-2 mediates mitotic defects in neural progenitors. Neuron 101, 1089-1098.e4 (2019).

101. Mesci, P. et al. Modeling neuro-immune interaction during Zika virus infection. Hum. Mol. Genet. 27, 41-52 (2018).

102. Muffat, J et al. Human induced pluripotent stem cell-derived glial cells and neural progenitors display divergent responses to Zika and dengue infections. Proc. Natl Acad. Sci. USA 115, 7117-7122 (2018)

103. Quicke, K. M. et al. Zika virus infects human placental macrophages Cell Host Microbe 20, 83-90 (2016).

104. Vermillion, M. S. et al. Intrauterine Zika virus infection of pregnant immunocompetent mice models transplacental transmission and adverse perinatal outcomes. Nat. Commun. 8, 14575 (2017).

105. Ginhoux, F. \& Guilliams, M. Tissue-resident macrophag ontogeny and homeostasis. Immunity 44, 439-449 (2016).

106. Puschnik, A. S., Majzoub, K., Ooi, Y. S. \& Carette, J. E. A CRISPR toolbox to study virus-host interactions. Nat. Rev Microbiol 15, 351-364 (2017).

107. Li, Y. et al. Genome-wide CRISPR screen for Zika virus resistance in human neural cells. Proc. Natl Acad. Sci. USA 116, 9527-9532 (2019).

This study highlights the potential to use hPSC based platforms to perform whole-genome screens in human, disease-relevant cells, in this case identifying critical host factors involved in ZIKV infection.

108. Xu, M. et al. Identification of small-molecule inhibitors of Zika virus infection and induced neural cell death via a drug repurposing screen. Nat. Med. 22, 1101-1107 (2016)

109. Zhou, T. et al. High-content screening in hPSC-neural progenitors identifies drug candidates that inhibit Zika virus infection in fetal-like organoids and adult brain. Cell Stem Cell 21, 274-283.e5 (2017).

Xu et al. (2016) and Zhou et al. (2017) provide examples of how hPSC-based models are scalable so that they can be used to perform high-content drug screens, here discovering therapeutic candidates that act against ZIKV infection.

110. Wilson, A. C. \& Mohr, I. A cultured affair: HSV latency and reactivation in neurons. Trends Microbiol. 20, 604-611 (2012).

111. Markus, A., Lebenthal-Loinger, I., Yang, I. H. Kinchington, P. R. \& Goldstein, R. S. An in vitro model of latency and reactivation of varicella zoster virus in human stem cell-derived neurons. PLoS Pathog. 11 e1004885 (2015).

112. Sadaoka, T. et al. In vitro system using human neurons demonstrates that varicella-zoster vaccine virus is impaired for reactivation, but not latency. Proc. Nat Acad. Sci. USA 113, E2403-E2412 (2016).

113. Pourchet, A., Modrek, A. S., Placantonakis, D. G. Mohr, I. \& Wilson, A. C. Modeling HSV-1 latency in human embryonic stem cell-derived neurons. Pathogens 6, 24 (2017)

Markus et al. (2015), Sadaoka et al. (2016) and Pourchet et al. (2017) apply hPSC-derived neurona cultures to study viral latency.

114. Tangye, S. G. et al. Human inborn errors of immunity: 2019 update on the classification from the international union of immunological societies expert committee. J. Clin. Immunol. 40, 24-64 (2020).

115. Hoffmann, M. et al. Chloroquine does not inhibit infection of human lung cells with SARS-CoV-2. Nature 585, 588-590 (2020)

116. Wang, M. et al. Remdesivir and chloroquine effectively inhibit the recently emerged novel coronavirus (2019-nCoV) in vitro. Cell Res. 30, 269-271 (2020).

117. Liu, J. et al. Hydroxychloroquine, a less toxic derivative of chloroquine, is effective in inhibiting SARS-CoV-2 infection in vitro. Cell Discov. 6, 16 (2020).

118. Gupta, A. et al. Extrapulmonary manifestations of COVID-19. Nat. Med. 26, 1017-1032 (2020)

119. Ellul, M. A et al. Neurological associations of COVID-19. Lancet Neurol. 19, 767-783 (2020)

120. Lamers, M. M. et al. SARS-CoV-2 productively infects human gut enterocytes. Science 369, 50-54 (2020).

121. Zhou, J. et al. Infection of bat and human intestinal organoids by SARS-CoV-2. Nat. Med. 26, 1077-1083 (2020).

122. Duan, X. et al. Identification of drugs blocking SARS-CoV-2 infection using human pluripotent stem cell-derived colonic organoids. Preprint at bioRxiv https://doi.org/10.1101/2020.05.02.073320 (2020).

123. Pérez-Bermejo, J. A et al. SARS-CoV-2 infection of human iPSC-derived cardiac cells predicts novel cytopathic features in hearts of COVID-19 patients. Preprint at bioRxiv https://doi.org/10.1101/2020.08. 25.265561 (2020)

124. Yang, L. et al. A human pluripotent stem cell-based platform to study SARS-CoV-2 tropism and model virus infection in human cells and organoids. Cell Stem Cell 27, 125-136.e7 (2020).

This study is the first to report the selective tropism of SARS-CoV-2 for specific subpopulations of CNS cells.

125. Sharma, A. et al. Human iPSC-derived cardiomyocytes are susceptible to SARS-CoV-2 infection. Cell Rep. Med. 1, 100052 (2020).

126. Monteil, V. et al. Inhibition of SARS-CoV-2 infections in engineered human tissues using clinical-grade soluble human ACE2. Cell 181, 905-913.e7 (2020)

127. Han, Y. et al. Identification of candidate COVID-19 therapeutics using hPSC-derived lung organoids. Preprint at bioRxiv https://doi.org/10.1101/2020.05. 05.079095 (2020)

128. Jacob, F. et al. Human pluripotent stem cell-derived neural cells and brain organoids reveal SARS-CoV- 2 neurotropism predominates in choroid plexus epithelium. Cell Stem Cell S1 934-5909, 30463-X (2020).

This is early example of using regionalized brain organoids to model SARS-CoV-2 tropism, establishing susceptibility of choroid plexus organoids for SARS-CoV-2 infection.

129. Song, E. et al. Neuroinvasion of SARS-CoV-2 in human and mouse brain. Preprint at bioRxiv https://doi.org/ 10.1101/2020.06.25.169946 (2020).

130. Topol, E. J. COVID-19 can affect the heart. Science 370, 408-409 (2020).

131. Dixit, A. et al. Perturb-seq: dissecting molecular circuits with scalable single-cell RNA profiling of pooled genetic screens. Cell 167, 1853-1866.e 17 (2016) 
132. Desole, G. et al. Modelling neurotropic flavivirus infection in human induced pluripotent stem cell-derived systems. Int. J. Mol. Sci. 20, (2019).

133. Lang, J. et al. An hPSC-derived tissue-resident macrophage model reveals differential responses of macrophages to ZIKV and DENV infection. Stem Cell Rep. 11, 348-362 (2018).

134. Cosset, É. et al. Human three-dimensional engineered neural tissue reveals cellular and molecular events following cytomegalovirus infection. Biomaterials $\mathbf{5 3}$, 296-308 (2015).

135. Markus, A. et al. Varicella-zoster virus (VZV) infection of neurons derived from human embryonic stem cells: direct demonstration of axonal infection, transport of VZV, and productive neuronal infection. J. Virol. 85, 6220-6233 (2011)

136. Kurapati, S. et al. Role of the JNK pathway in varicellazoster virus lytic infection and reactivation. J. Virol. 91, 00640 (2017).

137. Lee, K. S. et al. Human sensory neurons derived from induced pluripotent stem cells support Varicella-Zoster virus infection. PLOS ONE 7. e53010 (2012).
138. Sison, S. L. et al. Human cytomegalovirus disruption of calcium signaling in neural progenitor cells and organoids. J. Virol. 93, e00954-e01019 (2019).

139. Poole, E. et al. An iPSC-derived myeloid lineage model of herpes virus latency and reactivation. Front. Microbiol. 10, 2233 (2019).

140. D'Aiuto, L. et al. Human induced pluripotent stem cell-derived models to investigate human cytomegalovirus infection in neural cells. PLOS ONE 7, e49700 (2012).

141. Brown, R. M. et al. Human cytomegalovirus compromises development of cerebral organoids. J. Virol. 93, 00957-01019 (2019).

142. Sundaramoorthy, V. et al. Modelling lyssavirus infections in human stem cell-derived neural cultures. Viruses 12, 359 (2020).

143. Carty, M., Reinert, L., Paludan, S. R. \& Bowie, A. G Innate antiviral signalling in the central nervous system. Trends Immunol. 35, 79-87 (2014).

144. Guo, Y. et al. Herpes simplex virus encephalitis in a patient with complete TLR3 deficiency: TLR3 is otherwise redundant in protective immunity. J. Exp. Med. 208, 2083-2098 (2011).

\section{Acknowledgements}

O.H. and L.S are supported by grants from the US National Institutes of Health. O.H. was supported by a training award from the NYSTEM grant \#C32559GG and the Center for Stem Cell Biology at MSK. The authors thank all members of the Studer laboratory for their critical input and stimulating scientific discussions.

Author contributions

The authors contributed equally to all aspects of the article.

Competing interests

The authors declare no competing interests.

Peer review information

Nature Reviews Immunology thanks M. Deleidi and the other, anonymous, reviewer(s) for their contribution to the peer review of this work.

Publisher's note

Springer Nature remains neutral with regard to jurisdictional claims in published maps and institutional affiliations.

(c) Springer Nature Limited 2021 